\section{SAT0451 DISEASE COURSE PATTERNS IN SYSTEMIC LUPUS ERYTHEMATOSUS}

K. Tselios, D. Gladman, Z. Touma, J. Su, N. Anderson, M. Urowitz. Rheumatology, Toronto Western Hospital, University of Toronto, Toronto, Canada

Background: Previous studies described three patterns of disease activity over time in systemic lupus erythematosus (SLE), namely long quiescent, relapsing remitting and persistently active. However, they enrolled prevalent patients, many of whom in the late stages of the disease. As such, the patterns of disease course since diagnosis are not known.

Objectives: The aim of the present study was to assess the prevalence and characteristics of such patterns over 10 years of follow-up in an inception cohort.

Methods: The inception patients of a large lupus cohort (enrolled within 18 months of diagnosis, $n=883$ ), with at least 10 years of follow-up and no time interval >18 months between consecutive visits, were investigated. Monophasic (M) pattern was defined as a clinical SLEDAI-2K=0 [serology (anti-dsDNA antibodies and $\mathrm{C} 3 / \mathrm{C} 4$ levels) excluded], achieved within five years since enrollment and maintained for $\geq 10$ years after that. Relapsing-remitting (RR) pattern was defined based on $\geq 2$ remission periods (a remission period equals two consecutive visits with a clinical SLEDAI-2K=0), while patients with no remission were categorised as persistently active (PA). Descriptive and regression analyses were used to compare the different groups regarding cumulative damage at 10 years, mortality and flare rate beyond 10 years.

Results: Of 267 patients who fulfilled the inclusion criteria, $27(10.1 \%)$ were monophasics, 180 (67.4\%) RR and 25 (9.4\%) PA. Thirty-five patients (13.1\%) had only one remission period ("hybrid"). There were no significant differences regarding the demographic, clinical, immunological and therapeutic characteristics among groups at enrollment. At 10 years, PA patients had received significantly more glucocorticosteroids [39.4 $\pm 24.3 \mathrm{~g}$ vs. $16.6 \pm 10.7 \mathrm{~g}$ and $27.3 \pm 18.4 \mathrm{~g}$ for the $\mathrm{M}$ and RR groups, $\mathrm{p}<0.001$ ] and accumulated significantly more damage [SLICC/ $\mathrm{DI}=2.36 \pm 1.6$ vs. $0.93 \pm 1.07$ and $1.22 \pm 1.33$, respectively, $\mathrm{p}<0.001]$. A trend for higher mortality was observed in the PA patients [24\% vs. $13.3 \%$ and $11.1 \%$ for the $R R$ and $M$ patients respectively, $p=0.22]$ whereas death occurred earlier [18.4 \pm 4.4 vs. $20.1 \pm 7.2$ and $24 \pm 4.1$ years, $p=0.11$ ]. Beyond 10 years, the annual flare rate was higher for the $\mathrm{PA}$ and $\mathrm{RR}$ patients as compared to the monophasics $[0.50 \pm 0.40$ vs. $0.47 \pm 0.57$ vs. $0.10 \pm 0.18$ respectively, $\mathrm{p}<0.001]$. Multinomial regression analysis for group membership showed Black race [OR=2.78, 95\% $\mathrm{Cl}=1.05-7.31, \mathrm{p}=0.039]$ and higher adjusted mean SLEDAI-2K over the first two years $[\mathrm{OR}=1.21,95 \% \mathrm{Cl}=1.11-1.32, \mathrm{p}<0.0001]$ to be associated with a more severe disease course.

Conclusions: Approximately $70 \%$ of lupus patients followed a relapsing remitting course from diagnosis onwards, while $10 \%$ displayed a monophasic and another $10 \%$ a persistently active course. Black race and more severe disease over the first two years were associated with a worse disease course.

Disclosure of Interest: K. Tselios: None declared, D. Gladman: None declared, Z. Touma: None declared, J. Su: None declared, N. Anderson: None declared, M. Urowitz Consultant for: Consultant GlaxoSmithKline

DOI: 10.1136/annrheumdis-2018-eular.5680

\section{SAT0452 ENDOTHELIUM DYSFUNCTION IN PATIENTS WITH SYSTEMIC LUPUS ERYTHEMATOSUS WITH AND WITHOUT NEPHRITIS}

N. Mohannad $^{1}$, A.I. El Zawawy ${ }^{2}$, M. Elbordeny ${ }^{3}$, D. Emara ${ }^{4}$, S. Ibrahim ${ }^{5} .{ }^{1}$ Internal Medicine department, Rheumatology Unit, Alexandria University Hospitals ${ }^{2}$ Internal Medicine department, Rheumatology Unit, ${ }^{3}$ Department of Clinical and Chemical Pathology, ${ }^{4}$ Department of Radiodiagnosis, Alexandria University;

${ }^{5}$ Internal Medicine, Ministry of Health, Alexandria, Egypt

Background: Organ-specific manifestations in systemic lupus erythaematosus (SLE) are highly influenced by the inherent characteristic of the vasculature ${ }^{(1)}$ Endothelial dysfunction might have significant roles in the pathogenesis of glomerular diseases.CD31 or platelet endothelial cell adhesion molecule-1 is known to have roles in angiogenesis, platelet function, thrombosis, and regulation of leukocyte migration through vascular walls ${ }^{(2)}$

Objectives: to assess the endothelium dysfunction in SLE patients (pts) and to determine its association with lupus nephritis (LN)

Methods: Study included 60 pts with SLE fulfilling the SLICC criteria for classification of SLE: 30 with(LN),30 SLE without LN and 10 matched healthy controls. Routine laboratory tests, lipid profile,renal functions, titres of autoantibodies [ANA anti ds-DNA, anticardiolipin antibodies $A C L(\lg M$, $\lg$ (g), flowcytometry to detect PECAM-1/CD31, doppler ultrasound to detect FMD of the brachial artery and SLEDAI were done to all SLE pts.Pts with diseases known to affect endothelial function:smoking,DM,essential HTN,known CAD were excluded

Results: Correlation between FMD\% and different parameters are shown in table 1.There was a statistical significant positive relation between CD31 and urea in $L N(p=0.014)$, non $L N(p=0.014)$, total SLE $p t s(p<0.001)$, creatinine in non $L N$ group $(p=0.025), 24$ hour protein in $\mathrm{LN}(\mathrm{p}<0.001)$, non $\mathrm{LN}(\mathrm{p}<0.001)$ and total SLE pts ( $p<0.001)$, cholesterol in $\mathrm{LN}(\mathrm{p}<0.001)$, non $\mathrm{LN}(\mathrm{p}=0.001)$ and total SLE pts $(p<0.001)$, LDL in $\operatorname{LN}(p=0.001)$, non $\operatorname{LN}(p=0.009)$ and total SLE $p t s(p<0.001)$,TG in total pts $(p=0.048)$, anti-dsDNA in $L N(p=0.024)$ and total SLE $p t s(p=0.008)$,SLE DAI in LN $(p=0.001)$ and total SLE $p t s(p<0.001)$.

\begin{tabular}{|c|c|c|c|c|c|c|}
\hline & \multicolumn{6}{|c|}{ FMD\% } \\
\hline & \multicolumn{2}{|c|}{ LN } & \multicolumn{2}{|c|}{ Non LN } & \multicolumn{2}{|c|}{ Total patients } \\
\hline & $\mathbf{r}$ & $\mathbf{p}$ & $\mathbf{r}$ & $\mathbf{p}$ & $\mathbf{r}$ & $\mathbf{p}$ \\
\hline \multicolumn{7}{|l|}{ Demographic data } \\
\hline Age & 0.033 & 0.862 & 0.275 & 0.141 & 0.057 & 0.666 \\
\hline D. duration & $-0.730^{*}$ & $<0.001^{*}$ & $-0.574^{\star}$ & $0.001^{*}$ & $-0.332^{*}$ & $0.010^{*}$ \\
\hline \multicolumn{7}{|l|}{ Renal function } \\
\hline Urea & $-0.771^{*}$ & $<0.001^{*}$ & $-0.689^{*}$ & $<0.001^{\star}$ & $-0.731^{*}$ & $<0.001^{*}$ \\
\hline Creatinine & -0.084 & 0.657 & 0.196 & 0.300 & $-0.324^{*}$ & $0.012^{*}$ \\
\hline Creatinine clearance & 0.084 & 0.660 & -0.024 & 0.898 & 0.200 & 0.126 \\
\hline $\begin{array}{l}24 \text { hour Urine protein } \\
\text { Lipid profile }\end{array}$ & $-0.543^{*}$ & $0.002^{*}$ & $-0.392^{*}$ & $0.032^{*}$ & $-0.631^{*}$ & $<0.001^{*}$ \\
\hline Cholesterol & $-0.438^{*}$ & $0.016^{*}$ & $-0.534^{*}$ & $0.002^{*}$ & $-0.530^{*}$ & $<0.001^{*}$ \\
\hline LDL & $-0.837^{*}$ & $<0.001^{*}$ & -0.779 & $<0.001^{*}$ & $-0.766^{*}$ & $<0.001^{*}$ \\
\hline Triglyceride & -0.252 & 0.179 & 0.086 & 0.652 & $-0.468^{*}$ & $<0.001^{*}$ \\
\hline \multicolumn{7}{|l|}{ Immunological profile } \\
\hline Anti DNA & $-0.517^{\star}$ & $0.003^{*}$ & $-0.648^{*}$ & $<0.001^{\star}$ & $-0.565^{*}$ & $<0.001^{*}$ \\
\hline SLEDAI & $-0.792^{*}$ & $<0.001^{*}$ & $-0.853^{*}$ & $<0.001^{*}$ & $-0.676^{*}$ & $<0.001^{*}$ \\
\hline
\end{tabular}

Fig 1 The performance of FMD to detect LN was better than anti-dsDNA and than CD31, where FMD was $80 \%$ sensitive and $100 \%$ specific at cut off point $>189>49 \%$

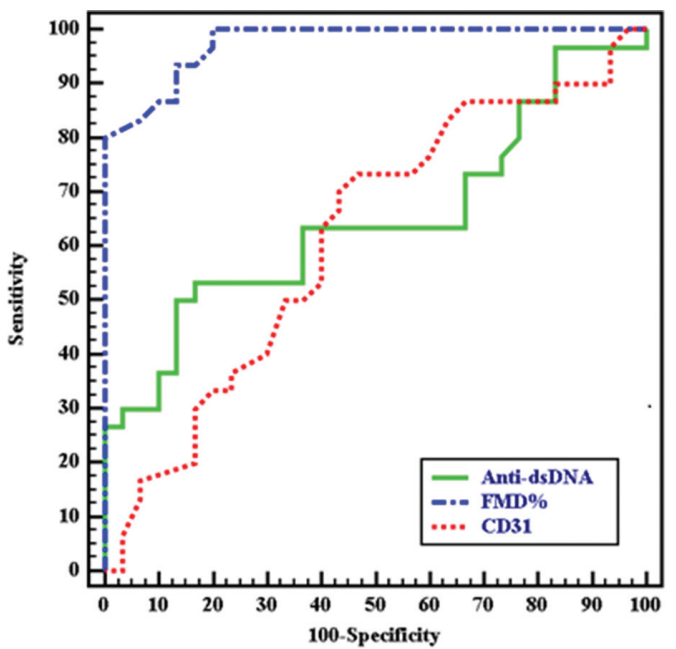

ROC curve for anti-dsDNA, CD31 \% and FMD \% to detect LN in SLE pts

Abstract SAT0452 - Figure 1. ROC curve for anti-dsDNA, CD31\% and FMD\% to detect LN in SLE pts

Conclusions: Endothelial dysfunction is the first step in the atherogenic process FMD and CECs may be used as markers for endothelial dysfunction in SLE. FMD correlated with proteinuria and may be used as an early marker for LN.CECs may play a role in atherosclerosis, vasculitis and LN

\section{REFERENCES:}

[1] Sesin CA, et al. Shedding of endothelial protein $C$ receptor contributes to vasculopathy \& renal injury in lupus: in vivo and in vitro evidence. Kidney Int 2005.

[2] Woodfi $n$ A, et al. PECAM-1: a multi-functional molecule in inflammation \& vascular biology. Arterioscler Thromb Vasc Biol 2007.

Disclosure of Interest: None declared

DOI: 10.1136/annrheumdis-2018-eular.4465 